Cuadernos de Filología Italiana

ISSN: 1133-9527

http://dx.doi.org/10.5209/CFIT.56038

\title{
La poesia testimoniale di Frabotta
}

\author{
Carmelo Princiotta ${ }^{1}$
}

Recibido: 08 de abril de 2017 / Aceptado: 30 de mayo de 2017

Riassunto. Il contributo interpreta le nuove poesie di Biancamaria Frabotta in una prospettiva testimoniale. Il trittico, che anticipa il prossimo libro, La materia prima, costituisce probabilmente l'avvio di un quarto tempo nella carriera dell'autrice. La senescenza si confronta con l'adolescenza del libro d'esordio, Il rumore bianco (1982), la testimone con l'«epoca muta e ciarliera», in un gioco a specchio fra passato e presente che, attraverso l'esperienza e il pensiero, medita sulla funzione della poesia nel nostro tempo.

Parole chiave: Frabotta; poesia testimoniale; poesia italiana contemporanea.

\section{[en] The Frabotta's Testimonial Poetry}

\begin{abstract}
The purpose of this paper is to analyze three new poems by Biancamaria Frabotta as testimonial poetry. The triptyque (a preview of Frabotta's next book, La materia prima) is probably the starting point of a fourth stage in the poet's career. In these poems senescence confronts adolescence, the subject of Frabotta's first book, Il rumore bianco (1982), while the poet as witness confronts the present («a mute and chatty time»). The result is the interplay between past and present, between intellect and experience that becomes a meditation about poetry's role in our age.

Keywords: Frabotta; Testimonial Poetry; Contemporary Italian Poetry.
\end{abstract}

Sommario: 1. La materia prima: un quarto tempo? 2. L'epoca e la testimone 3. La conoscenza notturna delle cose 4. La penultima parola dei poeti 5 . Riferimenti bibliografici.

Come citare: Princiotta, Carmelo (2017): «La poesia testimoniale di Frabotta», Cuadernos de Filología Italiana, 24, pp. 249-256.

\section{Biancamaria Frabotta}

TRE POESIE ${ }^{2}$

da La materia prima

I

Per placare nelle ossa il bruciore

il crepitìo dei neuroni inceneriti

\footnotetext{
1 Sapienza-Università di Roma, Piazzale Aldo Moro, 5, 00185 Roma (RM), Italia. carmelo.princiotta@uniroma1.it

2 Per gentile concessione dell'autrice. Si tratta di tre testi inediti di un volume di prossima pubblicazione.
} 
l'avanzare della falange virale e il sonno irrefrenabile che serra le pupille aprite la finestra nel fumoso sole d'inverno alle brezze della giovanile insonnia. Testimone di un'irrilevante ebbrezza nella tua ombra epoca muta e ciarliera m'accomodai - servizievole.

Ci dettasti il prologo noi mancammo l'epilogo.

Fortunata generazione senza assedio mi arriva dall'orto una voce riciclata e la serbo, come fossimo in guerra ed avessimo estirpato col granone sul prato il loglio e il nutrimento.

II

Plasma

sotto al sole non c'è

altro che il suo calore

Se ci acceca

è per non farsi frugare

per obbligarci a dire

"là c'è segreto".

L'accecato di primo mattino.

Quale migliore testimone

ai fasti della luce diurna

quando crolla l'attrito

che tiene uniti i gas

nell'immensa famiglia stellare?

L'agitazione termica scioglie

i legami e tanti gli urti subiti

che l'atomo s'apre a corolla

e se ne vanno in giro

nel cosmo i suoi pistilli

dove ogni sì ci plasma

e ogni no, in eguale misura.

Oh notte remissiva

fraterna spettatrice. 
III

Fede cruenta, arbitrio che non senti ragioni e dispensi il martirio incagliata tra i sassi e le scaglie del torrente vai verso l'inutile foce col passo adolescente e la voce della rincorsa vai con quel tuo miagolìo inudibile, infossando canali alle lacrime. E' l'era dei maestri gli ultimi testimoni sono stati arsi. Cura sulle guance la febbre l'avvento calmo di Galileo l'abiura ai piedi del Galileo lui che le prove le ha in tasca.

Ma per ogni verità non provata bisogna credere in compagnia della santa canaglia.

Fiore di bulbo intirizzito dal vento di marzo declini nel gelo la colpa della sopravvivenza inviandoci le tue sorelle, carità e speranza. A noi dopo ambite morti immortali giacché si muore solo una volta spetta la penultima tardiva parola.

\section{La materia prima: un quarto tempo?}

Biancamaria Frabotta anticipa qui, nella forma compatta di un trittico, versi di un libro a venire, annunciandone il titolo, semplice e potente: La materia prima. Ogni nuovo libro ha bisogno di una discontinuità rispetto al precedente e di una continuità con il resto dell'opera. Un poeta, specie d'età, può giungere a un punto della propria vicenda in cui si inoltra nell'altro versante dell'opera, passando magari dal dritto al rovescio o, per dirla con Montale, dal recto al verso, con esiti talora sorprendenti e, come nel caso di Satura, persino sconcertanti. Cambia il rapporto con il proprio corpo e con il proprio tempo: biologia e storia collaborano a quello stile tardo che può non di rado tradursi in maniera. Si entra nella zona della penultimità, della prima vecchiaia, e i poeti rischiano di diventare fatui o saccenti. Cambia, soprattutto, la voce, che è l'anima musicale di ogni corpus poetico: talora si assottiglia fino al più stridulo falsetto, talaltra si abbassa nella raucedine del parlato più plumbeo. È ancora presto per dirlo, ma Da mani mortali (2012) si configura per molti aspetti come il grande libro conclusivo di una prima fase in tre tempi della poesia di Frabotta, che a breve dovremmo vedere riunita in un volume mondadoriano. Il primo tempo va dalla plaquette d'esordio Affeminata (1976) a Il rumore bianco (1982): è il tempo turbolento della «seconda nascita» (Frabotta 1976: 38) negli anni della nuova sog- 
gettività romana e italiana e della risorgenza poetica oltre la crisi della tradizione e dell'avanguardia. Il secondo, avviato dalla plaquette di svolta Appunti di volo e altre poesie (1985), comprende La viandanza (1995) e Terra contigua (1999): è il tempo mediano del movimento fra nostalgia e trasformazione, che sfata precocemente $i$ falsi miti della globalizzazione attraverso la dialettica fra progresso e distruzione e propone senza presunzione un'uscita dal postmodernismo. Il terzo prende le mosse da La pianta del pane (2003) e probabilmente si chiude con Da mani mortali: è il tempo dell'intelligenza residenziale, che congeda come in un requiem personale e politico il Novecento e, dalla specola maremmana di Cupi, perlustra il presente nell'asimmetria della vita activa, secondo una prospettiva morale, ecocritica e, in fin dei conti, pagana.

Fin qui il recto dell'opera ${ }^{3}$. E dopo? Nel 2015 Frabotta ha pubblicato un manipolo di nuovi testi nella plaquette Per il giusto verso: un titolo che oggi possiamo leggere come un involontario augurio perché il verso dell'opera non ne tradisca il recto, come invece avviene nell'ultimo Montale. Un augurio inverato dalle tre poesie qui anticipate: si tratta di testi accomunati dalla dimensione testimoniale, come rivelano, anche a livello lessicale, i vv. 9, 6 e 12 dei rispettivi componimenti. Sono poesie di testimonianza e, insieme, poesie sulla testimonianza, come si addice a una scrittrice dell'esperienza e del pensiero quale è Frabotta.

\section{L'epoca e la testimone}

Nel primo testo, Per placare nelle ossa il bruciore, il testimone è l'io e si rapporta con l'epoca, offrendoci un mirabile autoritratto: «Testimone di un'irrilevante / ebbrezza nella tua ombra / epoca muta e ciarliera / m'accomodai — servizievole» (vv. 9-13). L'epoca è «la grande taciturna» di Anna Achmàtova (Achmàtova 1966: 103), il secolo tragico che abbiamo alle spalle, ma è anche il tempo della proliferazione dei discorsi, forse fino alla chiacchiera contemporanea, e la stagione di un'innumerevole ripresa di parola, dopo la rivoluzione culturale del Sessantotto. Vengono quindi evocate, con l'understatement di chi si confronta con i destini generali, l'ebbrezza poetica di quella stagione e la militanza vissuta come servizio. Il componimento si apre, però, con la descrizione di un corpo sotto assedio per un terribile attacco virale: un episodio di resistenza biologica che sembra modellato sull'assedio di Leningrado durante la seconda guerra mondiale e composto quasi in controcanto con il Poema senza eroe della grande russa. La storia diventa una condizione allegorica, un modo di interpretare l'ethos del proprio stare al mondo, come per una tragica coscienza intellettuale sedimentata nelle ossa della propria persona e della propria poesia. $\mathrm{Per}$ placare nelle ossa il bruciore è un testo di risveglio dal «sonno irrefrenabile» (v. 4),

\footnotetext{
Per una prima interpretazione dell'opera di Frabotta ci si può avvalere della monografia di Marco Corsi 2010). Oltre alla fondamentale rimessa a disposizione di libri da troppo tempo introvabili, attraverso un volume di Poesie, urgerebbe raccogliere gli interventi critici su Frabotta sparsi in miscellanee, riviste e quotidiani e la dispersa serie di interviste, interventi e autocommenti rilasciati da Frabotta nel corso degli anni. La difficoltà, diciamo pure montaliana, di alcuni luoghi testuali rende particolarmente auspicabile un'antologia commentata dalla stessa autrice, sul modello de Il figurante di Maurizio Cucchi (1985), o da studiosi, come per l'edizione di Ora serrata retinae di Valerio Magrelli (2012) curata da Sabrina Stroppa e Laura Gatti e per la sezione di Somiglianze di Milo De Angelis edita da Fabio Jermini (2015). Sulla necessità di un'edizione commentata de Il rumore bianco, per esempio, si è espresso di recente Rodolfo Zucco (2016: 200), augurandosi una più vasta operazione editoriale che riguardi i poeti degli anni Settanta e Ottanta.
} 
della degenza coatta, della reclusione sanitaria: l'apertura della finestra riporta «le brezze / dell'insonnia giovanile» (vv. 7-8), rianima la voce senescente. Insieme alla vita riaffluisce la poesia, che marezza la metrica con le prime inarcature del testo, il corrispettivo formale del risveglio. Ricompare l'irrequietezza sentimentale, che detta la vitalità musicante del verso. È un io che torna in sé, che torna a essere sé stesso: ed ecco che emerge il segno dell'identità stilistica di Frabotta, la paronomasia (brezze-ebbrezza, vv. 7-10), insieme a uno dei suoi temi dominanti: l'insonnia, sintomo di turbamento esistenziale e condizione di veglia critica. Tutta la scrittura di Frabotta è caratterizzata dalla tensione fra l'irrequietudine del sentimento e la fermezza del pensiero, con conseguente oscillazione fra formulazioni epigrammatiche, o comunque gnomiche, talora disposte a cornice, e un fraseggio emotivo diciamo pure scaleno, che in genere occupa la parte centrale del testo. Questa tensione, indice di leopardismo profondo prima ancora che di montalismo, non si realizza in strutture fisse, altrimenti meccaniche, ma costituisce la dinamica interna comune ai singoli individui testuali, veicolando l'opposizione fondamentale della scrittura frabottiana: quella fra la tragicità della coscienza intellettuale e la felicità dell'esperienza formale, che è poi la testimonianza antinichilista della poesia. Ed è ciò che scuote le certezze negative della ragione con i lampi dell'intelligenza emotiva. Questa opposizione si traduce in una gestione della compagine ritmica che ricorre ora a versi più lunghi ora a versi più brevi e mobilita i rapporti fra metro e sintassi, alternando scompensi ed equivalenze, e che, in fin dei conti, procede per scansioni meliche e scansioni discorsive. Per placare nelle ossa il bruciore è un risveglio alla vita che fa tutt'uno con il risveglio della poesia. Ebbene, questo secondo risorgimento innesca un raffronto col primo, quello del tempo d'esordio, «il risorgimento di narcisa» (Frabotta 1982: 38); stabilisce una frizione fra il nunc e il tunc che fa certo pensare alla polarità fra i due tempi di Poema senza eroe: il 1913 e l'assedio di Leningrado, interpretato da Achmàtova come «il rovescio della medaglia» (Achmàtova 1966: 103) sul cui dritto era iscritta l'arlecchinata avanguardistica del primo anteguerra. Un ulteriore raffronto si stabilisce, del resto, fra la generazione assediata dalla guerra e la «generazione senza assedio» (v. 15), che ha scontato una rovina immateriale, quella di un diffuso azzeramento culturale, destato come per contraccolpo dall'insorgenza di nuovi soggetti culturali, se interpreto bene il senso dei versi finali. È stata fatta tabula rasa, senza distinzione fra grano e zizzania: così siamo rimasti senza nutrimento. Come avviene con certe soluzioni mediche, che per sradicare il male da un organismo ne aggrediscono anche la parte sana.

\section{La conoscenza notturna delle cose}

Nel secondo testo, intitolato Plasma, il testimone è anonimo, «l'accecato di primo mattino» (v. 5), e sta al cospetto del sole evocato in epigrafe: «sotto al sole non c'è altro che il suo calore». È questa la chiave del componimento. A un primo livello, l'epigrafe ha una funzione referenziale: suggerisce che il sole è il soggetto sottinteso del periodo incipitario e che la sua attività è anche il plasma del sistema solare. A un secondo livello, però, l'epigrafe rimanda, senza rivelarlo, a Il rumore bianco: la frase, in realtà, è un verso di Mi stupisci di schiena e fra di noi (Frabotta 1982: 97). In questa sede non conta che quel verso arieggi un verso di Ingeborg Bachmann (Princiotta 2015: 895), ma che Frabotta stia rileggendo sé stessa e, in particolare, 
il libro d'esordio. Il corpo centrale della poesia è una straordinaria rivisitazione dell'entropia, il fenomeno fisico che aveva dettato la metafora poetica, esistenziale e storica de Il rumore bianco ${ }^{4}$. Il plasma è anche ciò che ci mantiene in vita dopo uno choc biologico, ci lascia sotto il sole, conservandoci al suo calore. Il titolo, dunque, potrebbe essere ambivalente, come sempre è il linguaggio della poesia, anche quando ricorre al linguaggio della scienza. Il plurilinguismo di Frabotta, renitente alle retoriche dell'orfismo e a quelle del semplicismo, mostra in tutta evidenza che la precisione del linguaggio poetico non ne elimina mai la costitutiva ambiguità, essenza stessa della sua alterità. Al v. 16 plasma, voce verbale, entra in attrito con il sostantivo del titolo: l'entropia ci forma, ci plasma, con i suoi urti particellari, fra aggregazioni e disgregazioni di materia. La stessa paronomasia, che, come si è detto, è la firma stilistica di Frabotta, non è altro che un corrispettivo retorico dell'entropia, una generatrice di urti verbali che plasma la materia della poesia ${ }^{5}$.

Forma e tema sono strettamente connessi in quest'opera, fin dal principio. Tornano qui, non perché l'autrice intenda proporre una poesia di secondo grado, che slabbri e spiattelli le forme e i temi di un tempo in un conversato mondano o, all'inverso, che li estremizzi, scarnificandoli, in filosofemi antimetafisici, ma perché il pensiero potenzi l'esperienza, in quella che è, semmai, una poesia della seconda volta, del giusto verso. Così il binomio contrastivo giorno-notte, che ne Il rumore bianco indicava la tensione fra l'ordine e il disordine, come pure fra il bisogno di storia e il bisogno di poesia, è qui ripreso per contrapporre due forme di conoscenza. Il testimone diurno celebra con il suo accecamento l'abbagliante grandezza del sole e mostra insieme l'insufficienza della nuda percezione, la necessità della conoscenza scientifica, che sola può scoprire il segreto della vita cosmica. Al testimone diurno si contrappone la spettatrice notturna, una poesia che intrattiene un rapporto solidale con la scienza, tanto nella contemplazione rapita e consapevole dell'universo quanto nella capacità di cogliere la verità umana dei fenomeni fisici. Non si fa poesia senza scienza, ma la scienza non annulla la poesia, che si presenta qui come una forma di conoscenza notturna, alternativa all'accecamento della percezione diurna.

\section{La penultima parola dei poeti}

Nel terzo testo, Fede cruenta, arbitrio, non c'è un testimone, «gli ultimi testimoni sono stati arsi» (v. 12), anche se aleggia invisibile la figura di Giordano Bruno, bruciato vivo perché non aveva le prove della pluralità dei mondi e doveva testimoniarla con il martirio. Con Galileo comincia l'«era dei maestri» (v. 11): l'abiura non smentisce la dimostrazione scientifica prodotta, rende superflua la testimonianza per le verità già provate. In filigrana, è possibile scorgere un passo di Testimoniare di Edoardo Ferrario ben presente a Frabotta:

$4 \quad$ L'epigrafe del libro d'esordio, tratta da Akiva Moiseevich Yaglom, recitava: «Per esempio consideriamo il moto casuale di una particella immersa in un fluido e la fluttuazione del numero di collisioni che le molecole del fluido hanno con la particella. Questa fluttuazione la chiamiamo "rumore bianco"».

5 Non va dimenticato che il testo di apertura di Affeminata e, quindi, dell'opera di Frabotta (1976: 5), Fra le piante dimentiche dei parti prematuri, ha come occasione per così dire contingente la lettura del De rerum natura di Lucrezio. 
Giordano Bruno ha testimoniato «fino all'ultimo» di una verità in cui non poteva che credere, dato che non era in grado di fornire alcuna prova del sistema copernicano. Non così Galileo, che l'ha dimostrato, provato. E forse è proprio questa dimostrazione che gli ha consentito di abiurare, di spergiurare, di ritrattare - e di salvarsi la vita. Per la verità della sua dimostrazione non c'era più bisogno di lui e della sua testimonianza. Era stato reso inutile, come testimone, dalla dimostrazione che aveva prodotto, dalla prova che aveva messo davanti a tutti e a chiunque poteva leggere e ripetere i passaggi dimostrati nel suo testo e nei suoi esperimenti. Galileo ha potuto essere un maestro senza il bisogno di essere un testimone e un martire. (Ferrario 2006: 24)

La chiusa del trittico è un testo sulla duplicità della fede: un'altra opposizione. C'è una fede dogmatica, confessionale, nemica della ragione e della scienza, dispensatrice di morte, e c'è una fede laica, necessaria a sostenere le verità indimostrate dei poeti, amica della ragione e della scienza, ma consapevole della loro se non altro provvisoria insufficienza. Viene così ripensato e riformulato, io credo, il mandato testimoniale dei poeti: «A noi dopo ambite morti immortali / giacché si muore solo una volta / spetta la penultima tardiva parola» (vv. 22-24). Non c'è nessuna presunzione vaticinante in questa affermazione, ma la necessità di assicurare un servizio alla comunità, in un mondo che può misconoscere il ruolo dei poeti ma non può impedire che continuino a esercitare la loro funzione: «Borderliners di una Verità in cui il divino non si riconosce, ai margini della polis, dove politici e sciamani si fronteggiano nella lotta per l'egemonia, i poeti restano sulla frontiera di una porta socchiusa, ospiti indesiderati, scomodi testimoni e, letteralmente, cittadini ex lege» (Frabotta 2009: 102). Per intendere le nuove poesie di Frabotta bisogna rileggere l'ultimo tempo di Quartetto per masse e voce sola, intitolato Il tempo a venire (Frabotta 2009: 95-118): preambolo più che commentario in prosa di questi versi.

\section{Riferimenti bibliografici}

Achmàtova, Anna (1966): Poema senza eroe e altre poesie, a cura di Carlo Riccio, Torino, Einaudi, pp. 56-121.

Ferrario, Edoardo (2006): Testimoniare, a cura di Franco Maria Fontana, Roma, Lithos.

Corsi, Marco (2010): Biancamaria Frabotta. I nodi violati del verso, con uno scritto di Maria Carla Papini, Bologna, Archetipo Libri.

Cucchi, Maurizio (1985): Il figurante (1971-1985), Firenze, Sansoni.

De Angelis, Milo (2015): «Intervallo e fine». Commento a una sezione di 'Somiglianze' (1976), a cura di Fabio Jermini, Lecce, Pensa Multimedia.

Frabotta, Biancamaria (1976): Affeminata, con una nota critica di Antonio Porta, Rivalba, Geiger.

Frabotta, Biancamaria (1982): Il rumore bianco, prefazione di Antonio Porta, Milano, Feltrinelli.

Frabotta, Biancamaria (1985): Appunti di volo e altre poesie (1982-1984), Roma, La Cometa. Frabotta, Biancamaria (1995): La viandanza (1982-1992), Milano, Mondadori, Milano.

Frabotta, Biancamaria (1999 e 2011): Terra contigua, Roma, Empirì.

Frabotta, Biancamaria (2003): La pianta del pane, Milano, Mondadori. 
Frabotta, Biancamaria (2009): Quartetto per masse e voce sola, Roma, Donzelli.

Frabotta, Biancamaria (2012): Da mani mortali, Milano, Mondadori.

Frabotta, Biancamaria (2015): Per il giusto verso, Lecce, Manni.

Magrelli, Valerio (2012): Ora serrata retinae (1980), a cura di Sabrina Stroppa e Laura Gatti, Torino, Ananke.

Princiotta, Carmelo (2015): Dante DNA della poesia? Etica e lingua dopo il '68, in in Patrizia Bertini Malgarini, Nicola Merola e Caterina Verbaro (a cura di), La funzione Dante e $i$ paradigmi della modernità, Atti del XVI Convegno Internazionale della MOD (Roma, LUMSA, 10-13 giugno 2014), Pisa, ETS, pp. 893-900.

Zucco, Rodolfo (2016): «Se / perché / chi / come commentare», in in Beatrice Manetti, Sabrina Stroppa, Davide Dalmas, Stefano Giovannuzzi (a cura di), Poesia '70-'80: le nuove generazioni. Geografia e storia, opere e percorsi, letture e commento, selezione di contributi dal Convegno (Torino, 17-18 dicembre 2015), Genova, San Marco dei Giustiniani, pp. 195207. 